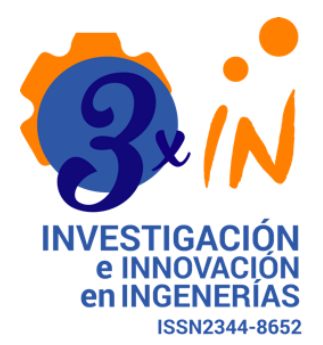

Open Access

Recibido:

10 octubre de 2018

Aceptado:

22 enero de 2019

Publicado:

1 julio de 2019

Correspondencia:

josnel.martinez@aunarcali.edu.co

jbarreto@col.luz.edu.ve

DOI:

https://doi.org/10.17081/invinno.7.2 .3448

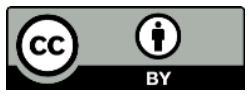

(c) Copyright: Investigación e Innovación en Ingenierías

\section{Modelo de planeación para la inversión tecnológica en centros de investigación universitarios}

\author{
Planning model for technological investment in university research \\ centers \\ Josnel Martínez Garcés \\ Corporación Universitaria Autónoma de Nariño - AUNAR Cali, Colombia \\ Javier Barreto Fereira \\ Instituto Universitario de Tecnología de Maracaibo, Venezuela
}

Resumen

Objetivo: Caracterizar el proceso de planeación de la inversión tecnológica en centros de investigación universitarios, específicamente, en los que integran el núcleo humanístico de la Universidad del Zulia (LUZ), Venezuela. Metodología: La investigación fue de tipo descriptiva con diseño de campo no experimental y de corte transeccional. Para recolectar los datos, se aplicó un cuestionario estructurado por 36 preguntas, dirigido a los directores de cada uno de los diecisiete (17) centros de investigación que componen el núcleo humanístico de la Universidad del Zulia (Venezuela). Por lo anterior, no fue necesario aplicar técnicas de muestreo sino el levantamiento de un censo poblacional. Resultados y Conclusiones: Los resultados permiten concluir que se hacen planes para la inversión tecnológica en todos los niveles de la estructura organizativa (estratégico, funcional y operativo), pero es necesario mejorar la comunicación de estos planes entre los diversos actores de los centros de investigación con el fin de garantizar su correcto seguimiento y efectivo cumplimiento.

Palabras claves: Centros de investigación, inversión, tecnología, planeación, universidades.

\begin{abstract}
Objective: This paper aims to characterize the planning process of technological investment in university research centers, specifically, in those that integrate the humanistic nucleus of Universidad de Zulia (LUZ), Venezuela. Methodology: A descriptive type with a nonexperimental design and a transactional data, was conducted. A questionnaire structured by 36 questions to the directors of each of the seventeen (17) research centers which belong to humanistic nucleus of Universidad de Zulia (Venezuela) was applied in order to collect the data; that is why, it was not necessary to apply sampling techniques but the lifting of a population census. Results and Conclusions: results allow to conclude that plans are made for technological investment in all levels of the organizational structure as follows: (strategic, functional and operational), but it is necessary to improve the communication process of these plans among different actors who are part of those research centers in order to guarantee their correct follow-up and effective compliance.
\end{abstract}

Keywords: Research Centers, investment, technology, planning, universities.

Como citar (IEEE): J. Martínez Garcés., y J. Barreto Fereira, “Modelo de planeación para la inversión tecnológica en centros de investigación universitarios", Investigación e Innovación en Ingenierías, vol. 7, n². 2, 2019. DOI: https://doi.org/10.17081/invinno.7.2.3448 


\section{Introducción}

Es evidente que la tecnología ha aportado beneficios incontables a la humanidad. Sus implicaciones comprenden desde las más grandes invenciones (como satélites espaciales capaces de conectar el mundo entero) hasta herramientas cotidianas como el teléfono celular (que también sirve para conexión con otros, independientemente de donde estén, gracias al uso de Internet, por ejemplo). De ahí que cada vez sean más los países y las organizaciones que invierten grandes cantidades de recursos financieros en las tecnologías emergentes.

En este sentido, según cifras publicadas por el Banco Mundial [1], los países del mundo pasaron de destinar el $2 \%$ de su Producto Interno Bruto (PIB) al gasto de investigación y desarrollo en el año 2004 , al 2,16 \% en el 2014. Para ese último año, un $17,1 \%$ de las exportaciones mundiales involucraron productos de alta tecnología, lo que equivale a un total de 2.147 billones de dólares. Sin duda estos datos son un reflejo del auge de la inversión tecnológica mundial en la época contemporánea.

En este marco, es cada vez más apremiante que haya personas que investiguen y desarrollen productos innovadores y tecnológicos en los países y organizaciones del mundo. De acuerdo con el Banco Mundial [1], para el año 2010, había 1270 investigadores por cada millón de personas en el mundo, quienes normalmente ejercen sus funciones en organismos particulares dedicados a tal fin y en instituciones de educación superior financiadas por los entes de los distintos gobiernos.

A pesar de ello, el caso de Venezuela parece ser otro: al estudiar, en efecto, las universidades como cuerpos hacedores de ciencia e investigación, los hallazgos son poco alentadores. La situación financiera actual de las universidades públicas puede llevar a un desgaste y parálisis paulatina de las actividades que se desarrollan en estos centros de estudio [2]. En las universidades públicas de Venezuela y Latinoamérica, la inversión en ciencia y tecnología ha sido históricamente ínfima y es una de las más bajas del planeta, debido a que nunca han contado con el adecuado respaldo financiero del Estado y de los sectores productivos [3].

Al parecer, esta situación se replica en las universidades públicas venezolanas. En la Universidad de Oriente (UDO), por ejemplo, solo se recibió un $25 \%$ de los recursos del presupuesto total solicitado al gobierno nacional para el año 2016 [4]. Y esta situación apresura un cierre técnico de las instituciones de educación superior al no contar con los recursos necesarios para su funcionamiento [5]. 
La Universidad del Zulia (LUZ) no ha sido la excepción, pues del total de presupuesto solicitado para el año 2016, solo se le aprobó poco menos del $25 \%$ [6]. Como se ve, es la academia entonces quien sufre las consecuencias de la incapacidad financiera de la institución [7], pues esta situación de crisis presupuestaria se extiende a su actividad investigativa. De ahí la importancia de una adecuada planificación para el aprovechamiento de los recursos. En este marco contextual, la presente investigación tuvo como objetivo caracterizar el proceso de planeación de la inversión tecnológica en centros de investigación universitarios, y específicamente en los que integran el núcleo humanístico de la Universidad del Zulia (Venezuela).

\section{Proceso de planeación}

El proceso de planeación se concibe como la herramienta que permite vincular el presente y el futuro en las relaciones de la empresa, por lo que constituye una parte fundamental del proceso de toma de decisiones. De esta manera, el concepto de planeación consiste básicamente en definir los objetivos a lograr y los medios para realizarlos, o, con otras palabras, en visionar una situación futura y trabajar para construirla [8]. Complementando este concepto, se puede afirmar que la planeación es el acto de determinar las metas de una empresa y los medios para lograrlo, introduciendo así dos conceptos medulares: metas y planes [9].

Son cuatro las razones por las cuales se debe planear en una organización [10]: (a) para proporcionar dirección a los gerentes y a los empleados; (b) para reducir los escenarios de incertidumbre; (c) para minimizar el desperdicio y la redundancia; y (d) para establecer los estándares de control. Sin embargo, la planeación presenta ventajas y desventajas. Algunos aspectos a favor de ella, es que permite distribuir de manera justa los recursos y los procesos. Asimismo, gracias a ella, los procedimientos se pueden mejorar continuamente, lo que su vez permite mejorar la organización. Por último, la planeación facilita la coordinación de los recursos humanos, ayuda a la dirección de la empresa, disminuye el riesgo frente a posibles contingencias, entre otros. Pero, por otro lado, también consume tiempo y recursos, y en muchos casos se convierte en un mecanismo de resistencia al cambio, generando paradigmas controversiales entre las unidades operativas [11].

En otro sentido, el proceso de planeación obedece a unas características que se constituyen en principios de esta, a saber: la racionalidad, la previsión, la universalidad, la integridad y la continuidad. En este sentido, la previsión ubica el proceso en la dimensión temporal, ya que todo plan lleva implícito una previsión de lo que puede ocurrir. Por su parte, la universalidad no es otra cosa que la amplitud de aplicación de la planeación en un determinado contexto, situación o proceso; al tiempo que la integridad guarda relación 
con la concentración de intereses comunes en la institución. Finalmente, la continuidad se refiere al ciclo de vida del plan [12].

Por todo lo anterior, la apropiada planeación de la inversión tecnológica tendrá como resultado la correcta selección de las tecnologías necesarias para satisfacer las demandas de la organización. Con frecuencia, en efecto, cuando la planeación tecnológica es apropiada se minimizan los costos y a su vez se optimizan las inversiones.

Cabe diferenciar entre meta y planeación: Una meta es el estado futuro que la organización trata de alcanzar; mientras que los planes son proyectos en los que se especifica la asignación de recursos, los programas de trabajo y otras acciones necesarias para el alcance de las metas [9]. Las organizaciones deben integrar en el Plan Estratégico sus estrategias tecnológicas y los cursos de acción necesarios para llevarlas a cabo. Teóricamente, se han formulado algunos modelos aplicables a la gestión de la tecnología y lo expuesto en este apartado se condensa en el llamado "Modelo de dirección estratégica de la tecnología", el cual comprende varias etapas: (a) análisis, diseño y formulación de objetivos; (b) medios de implantación; y (c) medios de control [13].

Así, los objetivos son los resultados deseados: fines, propósitos, intenciones, estados futuros, que se alcanzarán mediante la aplicación de esfuerzos y recursos. De modo que alcanzar una meta implica llevar a cabo una serie de objetivos de manera sucesiva e interconectada. En la medida de lo posible, estos deben ser cuantitativos, formularse en términos numéricos y estar asociados a indicadores específicos de desempeño como: cantidades, datos, nivel de calidad o magnitud de los recursos empleados [8].

Por otro lado, los medios de realización definen el camino a seguir y se ponen en práctica por medio de actividades que movilizan la energía humana y los recursos de tiempo, espacio y equipos, entre otros, considerando la previsión de los recursos y actividades que se deben realizar para alcanzar la meta [8]. La organización no puede establecer objetivos imposibles de lograr a partir de los recursos disponibles. Es decir, las metas pueden ser desafiantes, pero también deben ser realistas. A fin de cuentas, por más deseos que se tengan de cumplir un objetivo, si no se cuenta con los medios necesarios para su realización, este resultará inviable [10].

Finalmente, los medios de control consisten en información que sirve para evaluar hasta qué punto se están alcanzando las metas y hasta qué punto son apropiados los cursos de acción [8]. A modo de resumen, el logro de los objetivos planteados conduce a alcanzar las metas de la organización; y estas últimas se desprenden de su misión y visión. Por su lado, los planes de acción son las rutas que, de manera articulada, se aplican para llegar a esas metas establecidas. 


\section{Tipos de planes}

A continuación se presentan los tipos de planes que se establecen dentro de las organizaciones en función de sus estructuras organizativas, a saber: planes estratégicos, planes funcionales y planes operativos [8].

Planes estratégicos: abarcan aspectos globales y amplios que ayudan a la organización a posicionarse en un largo plazo [14]. A través de ellos, se establecen los productos y servicios que se desean ofrecer, los mercados y clientes (internos y externos) que se desean atender, y las formas de lidiar con la competencia.

Definir los planes estratégicos es responsabilidad de la alta administración. En otras palabras, este tipo de planes se aplica a toda la empresa y a partir de ellos se establecen sus objetivos generales [10]. De esta manera, se convierten en la brújula orientadora de la organización y, por ende, de todos los procesos que integran su sistema.

Planes funcionales: son los que interpretan la planeación estratégica con el fin de acercarlos más a la realidad a través de planes específicos para el departamento o división [14]. Los planes funcionales abarcan áreas de actividades especializadas dentro de la organización, las cuales están claramente establecidas como apoyo a la estrategia [8]. Este tipo de planeación consiste en tomar decisiones concretas respecto a qué hacer, quién debe hacerlo y cómo debe hacerlo [15]. El correcto establecimiento de los planes funcionales permitirá determinar las rutas que se deben seguir para alcanzar lo planteado a través de la estrategia.

Planes operativos: especifican las actividades y los recursos que necesarios para realizar los objetivos. Aunque estos sean propios de la base de la pirámide organizacional, siempre hay un contenido operativo en cualquier tipo de plan [8], y dentro de lo operativo se encuentran las operaciones básicas que deben realizar los colaboradores [14]. Este tipo de plan suele ser mucho más limitado que los estratégicos y los funcionales. Asimismo, en cuanto a temporalidad, los planes pueden ser de corto plazo (cuando abarcan un período igual o menor a un año), de largo plazo (cuando abarcan un período mayor a tres años) o de mediano plazo (cuando abarcan un período mayor al de corto plazo pero menor al de largo plazo) [10].

Los planes operativos cumplen un rol importante en el alcance de los objetivos, debido a que se materializan lo planteado en los planes superiores $y$, generalmente, son los que demandan mayores recursos dentro de las organizaciones, tanto desde el punto de vista financiero como del humano. 


\section{Metodología}

Para el desarrollo del trabajo se aplicó una metodología descriptiva con un diseño de campo, no experimental y transeccional. La población estuvo integrada por los diecisiete (17) centros de investigación que conforman el núcleo humanístico de la Universidad del Zulia mostrados en la Tabla 1. Debido a su accesibilidad, no fue necesario realizar un muestreo, de manera que se levantó un censo poblacional.

Tabla 1. Distribución de la población

\begin{tabular}{|c|c|}
\hline № & Nombre del centro de investigación \\
\hline \multicolumn{2}{|r|}{ Facultad de Ciencias Económicas y sociales } \\
\hline 1 & Centro de Estadística e Investigación de Operaciones \\
\hline 2 & Centro de Estudios de la Empresa \\
\hline 3 & Centro de Estudios Sociológicos y Antropológicos \\
\hline 4 & Centro Socioeconómico del Petróleo y Energías Alternativas \\
\hline \multicolumn{2}{|r|}{ Facultad de Ciencias Jurídicas y Políticas } \\
\hline 5 & Centro de Investigaciones de Derecho Privado \\
\hline 6 & Centro de Investigaciones en Trabajo Social \\
\hline 7 & $\begin{array}{l}\text { Centro de Investigaciones y Estudios Laborales y de Disciplinas } \\
\text { Afines }\end{array}$ \\
\hline \multicolumn{2}{|r|}{ Facultad de Humanidades y Educación } \\
\hline 8 & Centro de Documentación e Investigación Pedagógica \\
\hline 9 & Centro de Estudios de la Actividad Física \\
\hline 10 & Centro de Estudios Filosóficos \\
\hline 11 & Centro de Estudios Geográficos \\
\hline 12 & Centro de Estudios Históricos \\
\hline 13 & Centro de Estudios Matemáticos y Físicos \\
\hline 14 & Centro de Investigación de la Comunicación y la Información \\
\hline 15 & Centro de Investigación en Química de los Productos Naturales \\
\hline 16 & Centro de Investigación y Desarrollo en Tecnologías del Conocimiento \\
\hline 17 & Centro de Investigaciones Biológicas \\
\hline
\end{tabular}

La institución fue seleccionada para el estudio debido a que es un referente académico en la región occidental de Venezuela; siendo de carácter público, gobierno autónomo y de importante influencia en la región. Los informantes clave fueron los directores de cada una de las referidas unidades de investigación. La observación de la variable se efectuó entre los meses de febrero y marzo del 2017.

Se recurrió a la encuesta como técnica de investigación y al cuestionario como instrumento para la recolección de datos. Este último estuvo integrado por 36 ítems, con opciones de respuesta dicotómicas en su primera fase exploratoria, así como por ítems con posibilidades de 
respuestas abiertas, y otras bajo la escala Likert, con el fin de añadirle profundidad y amplitud a la obtención de datos. Para el procesamiento de la información, se utilizó la estadística descriptiva para agrupar los elementos de acuerdo con su Frecuencia Absoluta (FA) y Frecuencia Relativa (FR). Una vez hecho este proceso, se construyeron las tablas que recogen los resultados.

\section{Resultados y discusión}

Para que la inversión tecnológica surta los efectos esperados en los procesos que desarrolla la organización, debe ser rigurosamente planificada. Por tal razón, el primer objetivo de esta investigación fue caracterizar el proceso de planeación de la inversión tecnológica en los centros de investigación del núcleo humanístico de la Universidad del Zulia. En este sentido, se encontró que la planeación está compuesta por los tres niveles típicos: nivel estratégico, nivel funcional y nivel operativo [8], pero con ciertas particularidades que enseguida se explican. Para cada uno de ellos se define un tipo de plan específico. En la Figura 1 se muestra de manera concreta el establecimiento de planes estratégicos para la inversión tecnológica en los centros de investigación del núcleo humanístico de la Universidad del Zulia.

Figura 1. Establecimiento de planes estratégicos para la inversión tecnológica en los centros de investigación del núcleo humanístico de la Universidad del Zulia

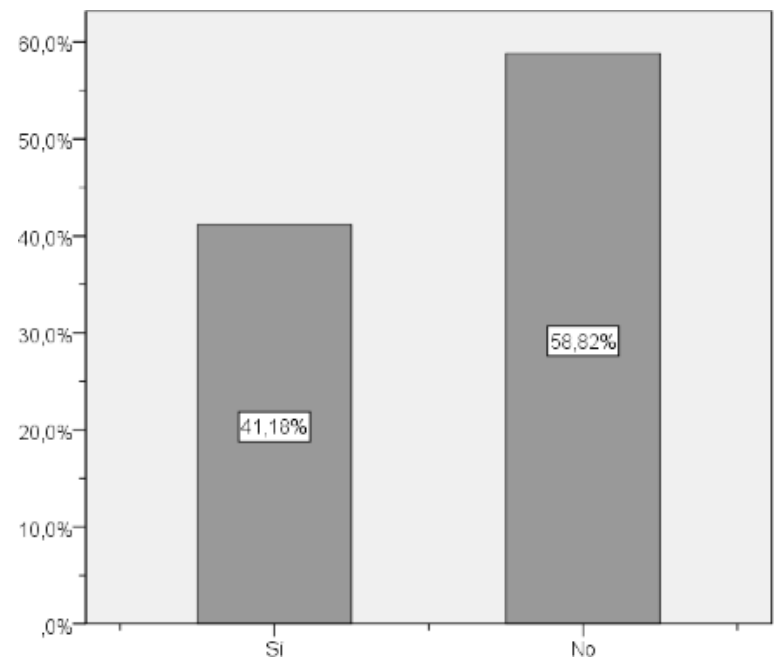

En relación con los planes estratégicos, o aquellos que son definidos por la alta administración a fin de fijar los objetivos generales [10], el Gráfico 1 muestra que sólo un 41,18 \% (siete de las unidades estudiadas) de los encuestados afirma que sí los establecen en relación a la inversión tecnológica, mientras que un 58,82 \% (diez de las unidades estudiadas) manifestó no hacer este tipo de planes en el nivel estratégico. En este orden de ideas, y con la intención de conocer cómo se gestionan los planes 
estratégicos para la inversión tecnológica en las unidades estudiadas, a continuación se presenta la Tabla 2.

Tabla 2. Gestión de los planes estratégicos para la inversión tecnológica en los centros de investigación del núcleo humanístico de la Universidad del Zulia

\begin{tabular}{|c|c|c|c|c|c|c|c|c|c|c|}
\hline \multirow{2}{*}{ Planes estratégicos } & \multicolumn{2}{|c|}{ Siempre } & \multicolumn{2}{|c|}{ Casi siempre } & \multicolumn{2}{|c|}{ Casi nunca } & \multicolumn{2}{|c|}{ Nunca } & \multicolumn{2}{|c|}{ Total } \\
\hline & $\mathrm{Fa}$ & Fr & $\mathrm{Fa}$ & $\mathrm{Fr}$ & $\mathrm{Fa}$ & $\mathrm{Fr}$ & $\mathrm{Fa}$ & $\mathrm{Fr}$ & $\mathrm{Fa}$ & $\mathrm{Fr}$ \\
\hline Se evalúa su cumplimiento & 2 & 28,57 & 4 & 57,14 & 1 & 14,29 & 0 & 0,00 & 7 & 10 \\
\hline $\begin{array}{l}\text { Se dan a conocer a los actores del nivel } \\
\text { funcional }\end{array}$ & 4 & 57,14 & 2 & 28,57 & 0 & 0,00 & 1 & 14,29 & 7 & $\begin{array}{c}10 \\
0\end{array}$ \\
\hline
\end{tabular}

Los valores de la Tabla 2 evidencian que los encuestados evalúan el cumplimiento de los planes estratégicos en inversión tecnológica así: un $28,57 \%$ lo hace siempre, un $14,29 \%$ lo hace casi siempre y un $14,29 \%$, casi nunca. A la pregunta sobre si se dan a conocer los planes estratégicos para inversión tecnológica a los actores del nivel funcional, un 57,14\% manifestó que siempre, un $28,57 \%$ que casi siempre, mientras que un $14,28 \%$ respondió no hacerlo nunca. Se comprueba, entonces, que es importante informar sobre los planes que se establecen desde el nivel estratégico dado que su ejecución tiene un efecto sobre toda la organización en general [10].

Pasando a los planes funcionales, es decir, a aquellos que posibilitan la realización de los planes estratégicos [8], se pudo conocer que un $64,71 \%$ (11 de las unidades estudiadas) de los encuestados los establece en relación con la inversión tecnológica, mientras que un 35,29\% (seis de las unidades estudiadas) restante no lo hace. Entre los centros de investigación que sí los establecen, un $41,21 \%$ manifestó que se derivaban de los planes estratégicos, mientras que el $23,50 \%$ restante manifestó que se desarrollan de manera empírica por parte de los actores de ese nivel.

Al contrastar estos resultados con la teoría que aborda el nivel de planeación al cual se hace referencia, se evidencia la importancia de establecer planes funcionales porque, desde la óptica de la inversión tecnológica, permiten abarcar áreas de actividades especializadas dentro de la organización [8]. En relación a esto, la Figura 2 recoge los resultados ya expresados sobre el establecimiento de planes estratégicos para la inversión tecnológica en los centros de investigación del núcleo humanístico de la Universidad del Zulia. 
Figura 2. Establecimiento de planes funcionales para la inversión tecnológica en los centros de investigación del núcleo humanístico de la Universidad del Zulia

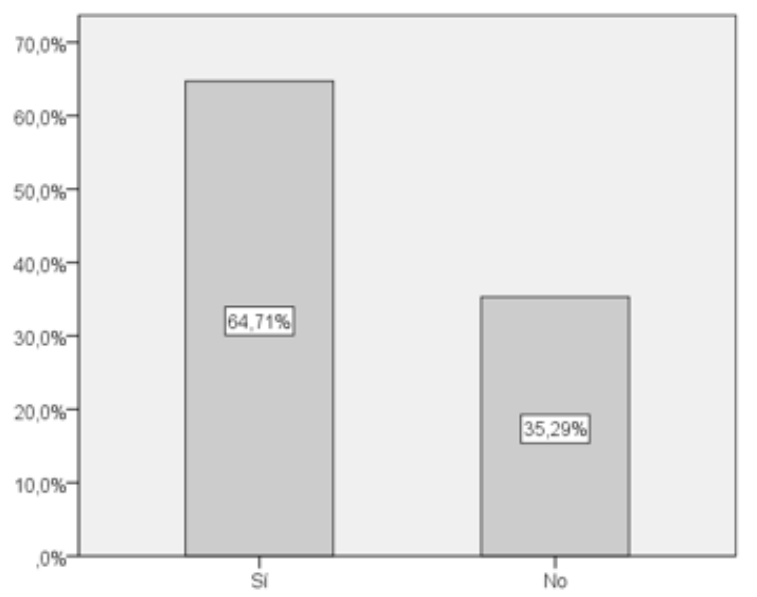

En este orden de ideas, la Figura 3 muestra la finalidad con la cual se establecen los planes funcionales para la inversión tecnológica en los centros de investigación del núcleo humanístico de la Universidad del Zulia.

Figura 3. Finalidad con la que se establecen los planes funcionales para la inversión tecnológica en los centros de investigación del núcleo humanístico de la Universidad del Zulia

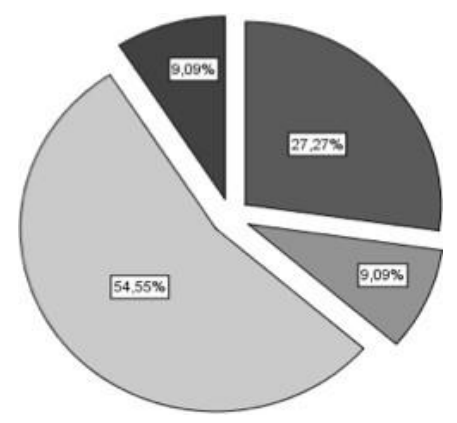

Como se ha dicho antes, los planes funcionales se establecen para decidir qué hacer, quién debe hacerlo y cómo [15]. Tomando esto en consideración, la Figura 3 evidencia que un $27,27 \%$ de los encuestados considera que los planes funcionales se establecen con la finalidad de definir qué tecnología adquirir, un 9,09\%, para definir cómo se debe adquirir la tecnología, un $54,55 \%$ lo hace con ambos fines, y el 9,09\% restante, por ninguna de las razones anteriores. Sobre este último porcentaje, es pertinente decir que la mayor parte está integrado por aquellos centros de investigación que no establecen planes de este tipo.

Asimismo, en este aspecto se quiso conocer cómo se gestionan los planes funcionales de inversión tecnológica en relación a los distintos niveles de 
planeación en los centros de investigación del núcleo humanístico de la Universidad del Zulia. Los resultados se recogen en la Tabla 3.

Tabla 3. Gestión de los planes funcionales para la inversión tecnológica en los centros de investigación del núcleo humanístico de la Universidad del Zulia

\begin{tabular}{|c|c|c|c|c|c|c|c|c|c|c|}
\hline \multirow{2}{*}{\begin{tabular}{c} 
Planes funcionales \\
\cline { 2 - 11 }
\end{tabular}} & \multicolumn{2}{|c|}{ Siempre } & \multicolumn{2}{|c|}{ Casi siempre } & \multicolumn{2}{|c|}{ Casi nunca } & \multicolumn{2}{|c|}{ Nunca } & \multicolumn{2}{|c|}{ Total } \\
\cline { 2 - 10 } $\begin{array}{c}\text { Se dan a conocer a los actores del nivel } \\
\text { operativo }\end{array}$ & 5 & 45,45 & 3 & 27,27 & 2 & 18,18 & 1 & 9,09 & $\begin{array}{c}1 \\
1\end{array}$ & $\begin{array}{c}10 \\
0\end{array}$ \\
\hline
\end{tabular}

La Tabla 3 muestra que un 45,45\% de los encuestados siempre da a conocer los planes funcionales a los actores del nivel operativo; un $27,27 \%$ lo hace casi siempre y un $18,18 \%$, casi nunca. Sin embargo, un 9,09\% manifestó nunca hacerlo. Al igual que en el nivel de planeación anterior, es claro que existen dificultades para comunicar los planes establecidos de un nivel a otro. Sin embargo, estos resultados demuestran nuevamente que un alto porcentaje de los encuestados que sí establecen planes funcionales se esfuerza por comunicarlos entre los distintos niveles de planeación, demostrando así la importancia de esta acción [10].

Finalmente, la Figura 4 muestra el establecimiento de los planes operativos para la inversión tecnológica en los centros de investigación del núcleo humanístico de la Universidad del Zulia.

Figura 4. Establecimiento de planes operativos para la inversión tecnológica en los centros de investigación del núcleo humanístico de la Universidad del Zulia

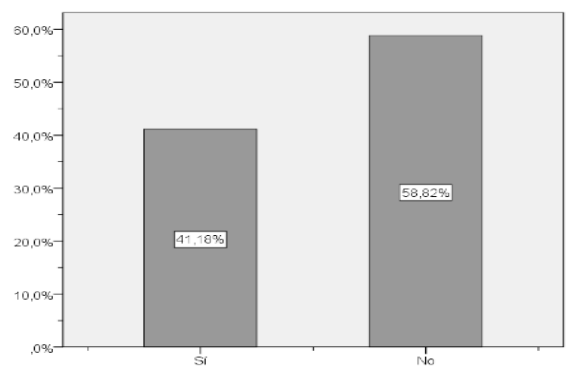

Los resultados expresados en el Figura 4 reflejan que sólo un 41,18\% (siete de las unidades estudiadas) de los encuestados afirma que sí establecen planes operativos en relación con la inversión tecnológica, mientras que un $58,82 \%$ (10 de las unidades estudiadas) manifestó no hacerlo. Tomando esto como base, se indagó sobre la forma en que se gestionan los planes funcionales; los resultados obtenidos se presentan en la Tabla 4. 
Tabla 4. Gestión de los planes operativos para la inversión tecnológica en los centros de investigación del núcleo humanístico de la Universidad del Zulia

\begin{tabular}{|c|c|c|c|c|c|c|c|c|c|c|}
\hline \multirow{2}{*}{ Planes operativos } & \multicolumn{2}{|c|}{ Siempre } & \multicolumn{2}{|c|}{ Casi siempre } & \multicolumn{2}{c|}{ Casi nunca } & \multicolumn{2}{|c|}{ Nunca } & \multicolumn{2}{|c|}{ Total } \\
\cline { 2 - 11 } & Fa & Fr & Fa & Fr & Fa & Fr & Fa & Fr & Fa & Fr \\
\hline $\begin{array}{c}\text { Dan a conocer las estrategias de } \\
\text { inversión tecnológica }\end{array}$ & 3 & 42,86 & 3 & 42,86 & 1 & 14,29 & 0 & 0,00 & 7 & $\begin{array}{c}10 \\
0\end{array}$ \\
\hline $\begin{array}{c}\text { Definen los bienes/recursos en los que } \\
\text { se invertirá }\end{array}$ & 5 & 71,43 & 1 & 14,29 & 1 & 14,29 & 0 & 0,00 & 7 & $\begin{array}{c}10 \\
0\end{array}$ \\
\hline
\end{tabular}

La Tabla 4 evidencia que, en general, los planes operativos dan a conocer las estrategias de inversión tecnológica en los centros de investigación del núcleo humanístico de la Universidad del Zulia, así: un $42,86 \%$ considera que esto ocurre siempre, un $42,86 \%$ que casi siempre y un $14,29 \%$, que casi nunca. Por otro lado, sobre si estos planes funcionales permiten definir los bienes o recursos tecnológicos en los cuáles se ha de invertir, un $71,43 \%$ considera que siempre lo definen, un $14,29 \%$ considera que casi siempre y el $14,29 \%$ restante, que casi nunca.

Esta información permite afirmar que estos son útiles para especificar las actividades y los recursos necesarios para realizar cualquier especie de objetivo, y aunque suela pensarse que esto ocurre sólo en el nivel estratégico, la realidad demuestra que siempre existe un contenido operativo en cada tipo de plan [8].

Finalmente, como valor agregado se buscó conocer el grado de relación existente entre los distintos niveles de planeación para la inversión tecnológica en los centros de investigación del núcleo humanístico de la Universidad del Zulia. Para ello se recurrió al uso de métodos estadísticos calculando el coeficiente de correlación Rho de Spearman. Los resultados obtenidos se muestran en la Tabla 5.

Tabla 5. Correlación entre los niveles de planeación para la inversión tecnológica en los centros de investigación del núcleo humanístico de la Universidad del Zulia

\begin{tabular}{|c|c|}
\hline $\begin{array}{c}\text { Relación entre los niveles de } \\
\text { planeación }\end{array}$ & $\begin{array}{c}\text { Rho de } \\
\text { Spearman }\end{array}$ \\
\hline Estratégico-Funcional & 0,495 \\
\hline Funcional-Operativo & 0,352 \\
\hline Operativo-Estratégico & 0,514 \\
\hline
\end{tabular}

Los datos contenidos en la Tabla 5 demuestran que entre los distintos coeficientes de correlación calculados se presenta una variación baja para 
cada relación de nivel de planeación. En este aspecto, todos los niveles poseen una correlación directa y positiva que oscila entre los rangos de "moderada" y "baja". Se destaca que el grado más alto de correlación se presenta entre los niveles operativo y estratégico con un coeficiente de 0,514 , demostrando estadísticamente que, entre más se establezcan los planes estratégicos para la inversión tecnológica, más moderadamente aumentan los planes operativos en este sentido.

Estos resultados en la correlación pueden deberse a que en un porcentaje considerablemente alto de los centros de investigación estudiados, los planes funcionales se establecen sin considerar los planes estratégicos e, incluso, en muchos casos de forma empírica. Por eso la relación solo predomina para la inversión tecnológica cuando ambos tipos de planes se establecen de manera acorde.

\section{Conclusión}

A través de los resultados de la presente investigación se concluye que el proceso de planeación de la inversión tecnológica en los centros de investigación del núcleo humanístico de la Universidad del Zulia se puede caracterizar de la siguiente manera:

\section{Planes estratégicos}

Menos de la mitad de los centros de investigación estudiados establece planes estratégicos en relación con la inversión tecnológica. Los que sí los establecen siempre hacen seguimiento de su cumplimiento. La gran mayoría se esfuerza por comunicar este tipo de planes a los actores del siguiente nivel, el funcional, aunque existe una minoría que no establece dicha comunicación.

El hecho de que algunos de estos centros de investigación reconozcan la importancia de establecer, evaluar y comunicar los planes estratégicos para la inversión tecnológica demuestra que reconocen su importancia y son conscientes de que su aplicación afecta a toda la estructura organizativa, a la vez que contribuyen al establecimiento de objetivos precisos.

\section{Planes funcionales}

La mayoría de los encuestados establece los planes funcionales en relación con la inversión tecnológica. El motivo por el que lo hacen es determinar qué tecnología adquirir, cómo adquirirla y quién debe adquirirla. En algunos casos estos planes se derivan de los planes estratégicos. Existe un número considerable de centros de investigación que manifestó desarrollarlos de manera empírica. 
La mayoría establece comunicación sobre estos planes funcionales para inversión tecnológica con los actores del siguiente nivel, el operativo, aunque existe una minoría que no lo hace.

Establecer planes funcionales para determinar el qué, el cómo y el quién de la tecnología a adquirir, así como la comunicación de los mismos a actores de otro nivel del centro de investigación sugiere que los encuestados reconocen que este tipo de planes permiten abarcar áreas especializadas de su estructura organizativa y pueden servir como apoyo para el cumplimiento de los planes estratégicos.

\section{Planes operativos}

Menos de la mitad de los encuestados los establece en relación a la inversión tecnológica. Este tipo de plan permite comunicar las estrategias concretas de inversión tecnológica y así definir en qué bienes o recursos se debe invertir. A pesar de que suelen considerarse mucho más limitados que los estratégicos o los funcionales, siempre hay un contenido operativo en cada tipo de plan. Al establecer este tipo de planes, los encuestados evidencian que reconocen su importancia para el cumplimiento de los objetivos establecidos en el centro de investigación.

Estos resultados permiten concluir, además, que en general sí existe una planeación de inversión tecnológica en los centros de investigación del núcleo humanístico de la Universidad del Zulia y que en ciertas ocasiones estos planes son evaluados para dar seguimiento a su cumplimiento. También queda claro que en algunos casos hay una apropiada comunicación entre los distintos niveles de planeación.

Asimismo, se evidencia estadísticamente que existe una correlación entre todos los niveles de planeación. La más destacada se presenta entre el nivel estratégico y nivel operativo, de manera que cuanto más se establezca uno, el otro aumenta moderadamente; no tratándose de una relación generada por causalidad. No obstante, para fortalecer esta planeación, se formulan los siguientes lineamientos:

Consolidar el establecimiento de planes estratégicos y de planes operativos para la inversión tecnológica en todos los centros de investigación como una política de gestión académico-administrativa, debido a que estos recaen sobre la alta administración [8].

Garantizar la adecuada y oportuna comunicación de los planes establecidos para la inversión tecnológica entre los diversos actores de los distintos niveles de cada unidad, ya que esto contribuirá en gran manera al cumplimiento de los objetivos generales [10]. 
Asegurar el establecimiento de planes funcionales para la inversión tecnológica que se deriven de los planes estratégicos, minimizando así el desarrollo empírico de los mismos. De este modo, se puede determinar qué hacer, quien debe hacerlo y como debe hacerlo [15].

\section{Referencias bibliográficas}

1. Banco Mundial. Gasto en investigación y desarrollo (\% de PIB)., 2016. Disponible en: www.datos.bancomundial.org

2. C. García, "Cecilia García: universidades pueden paralizarse por falta de presupuesto", Diario El Tiempo. 2016. Disponible en http://eltiempo.com.ve/venezuela/educacion/cecilia- garciauniversidades-pueden-paralizarse-por-falta-depresupuesto/211948

3. J. Aular, LUZ alerta sobre la poca inversión en la educación universitaria en América Latina. Vicerrectorado académico de la Universidad del Zulia., 2016. Disponible en:

http://www.viceacademico.luz.edu.ve/index.php?option=com_cont ent\&task=view\&id=795\&ltemid=202. Consultado el: 01/09/2016.

4. O. León, Vicerrector de la Unexpo Puerto Ordaz: "Todo está en muy malas condiciones", Correo del Caroní, 2016. Disponible en http://www.correodelcaroni.com/index.php/mas/educacion/item/ 44083- vicerrector-de-la-unexpo-puerto-ordaz-todo-esta-en-muymalas-condiciones

5. A. González, La universidad es muestra de cómo está el país. Diario El Tiempo, 2016.2 Disponible en http://eltiempo.com.ve/locales/barcelona/educacion/launiversidad-es-muestra-de-como-esta-venezuela/225042

6. J. Palencia, Presupuesto alcanza hasta marzo: LUZ a comisión de la AN. Noticia al día, 2016a. Disponible en http://noticiaaldia.com/2016/02/presupuesto-alcanza-hastamarzo-luz-a-comision-de-la-an/

7. J. Palencia, Rector Palencia: El presupuesto de funcionamiento de LUZ apenas está asegurado hasta marzo. Noticia al día, 2016b. Disponible en http://noticiaaldia.com/2016/03/rector-palencia-el-presupuestode-funcionamiento-de-luz-apenas-esta-asegurado-hasta-marzocomunicado/ Consultado el: 14/10/2016.

8. A. Amaru, Fundamentos de Administración. Teoría general y proceso administrativo. México: Editorial Pearson Prentice Hall, 2009.

9. R. Daft y D. Marcic, Introducción a la Administración. México: Editorial Thomson, 2006. 
10. S. Robbins y M. Coulter, Administración. México: Editorial Pearson Prentice Hall, 2010.

11. S. Hernández y Rodríguez, Administración. Pensamiento, procesos, estrategia y vanguardia. México: Editorial McGraw Hill, 2002.

12. J. Corredor, La planificación. Venezuela: Vadel Hermanos Editores, 2004.

13. C. Benavides y C. Quintana, “Dirección estratégica de la tecnología: el caso de Isofotón", Revista Universia Business Review, no. 8, pp. 68-85, 2005.

14. L. Boland, F. Carro, M. Stancatti, Y. Gismano y L. Banchieri, Funciones de la administración. Argentina: Editorial de la Universidad Nacional del Sur, 2007.

15. D. Hellriegel, S. Jackson y L. Slocum, Administración: un enfoque basado en competencias. México: Editorial Thompson, 2002. 